\title{
High power all-fiberized and narrow-bandwidth MOPA system by tandem pumping strategy for thermally induced mode instability suppression
}

\author{
Pengfei Ma ${ }^{1,2}, \mathrm{Hu} \mathrm{Xiao}^{1,2}{ }^{\text {, Daren Meng}}{ }^{1}$, Wei Liu ${ }^{1}$, Rumao Tao ${ }^{1,2}$, Jinyong Leng ${ }^{1,2}$, Yanxing Ma ${ }^{1,2}$, \\ Rongtao $\mathrm{Su}^{1,2}$, Pu Zhou ${ }^{1,2}$, and Zejin $\mathrm{Liu}^{1,2}$ \\ ${ }^{1}$ College of Advanced Interdisciplinary Studies, National University of Defense Technology, Changsha 410073, China \\ ${ }^{2}$ Hunan Provincial Collaborative Innovation Center of High Power Fiber Laser, National University of Defense Technology, \\ Changsha 410073, China \\ (Received 5 July 2018; revised 28 August 2018; accepted 28 September 2018)
}

\begin{abstract}
An all-fiberized and narrow-bandwidth master oscillator power amplification (MOPA) system with record output power of $4 \mathrm{~kW}$ level and slope efficiency of $78 \%$ is demonstrated. Tandem pumping strategy is tentatively introduced into the narrow-bandwidth MOPA system for thermally induced mode instability (TMI) suppression. The stimulated Brillouin scattering (SBS) effect is balanced by simply using one-stage phase modulation technique. With different phase modulation signals, SBS limited output powers of $336 \mathrm{~W}, 1.2 \mathrm{~kW}$ and $3.94 \mathrm{~kW}$ are respectively achieved with spectral bandwidths accounting for $90 \%$ power of $\sim 0.025,0.17$ and $\sim 0.89 \mathrm{~nm}$. Compared with our previous $976 \mathrm{~nm}$ pumping system, TMI threshold is overall boosted to be $>5$ times in which tandem pumping increases the TMI threshold of $>3$ times. The beam quality ( $M^{2}$ factor) of the output laser is well within 1.5 below the TMI threshold while it is ultimately saturated to be 1.86 with the influence of TMI at maximal output power. Except for SBS and TMI, stimulated Raman scattering (SRS) effect will be another challenge for further power scaling. In such a high power MOPA system, multidetrimental effects (SBS, SRS and TMI) will coexist and may be mutual-coupled, which could provide a well platform for further comprehensively investigating and optimizing the high power, narrow-bandwidth fiber amplifiers.
\end{abstract}

Keywords: advanced laser technology and applications; design; fiber laser and applications; high power laser; laser amplifiers; laser systems; modeling; narrow linewidth; optimization

\section{Introduction}

High power fiber laser sources with near-diffraction-limited beam quality have been strongly required in many scientific and industrial applications ${ }^{[1-3]}$. However, power scaling of the monolithic fiber laser system has been challenged as a result of the limitations of nonlinear effects, fiber damage, high brightness pump sources and thermally induced mode instability $(\mathrm{TMI})^{[4-6]}$. Coherent and spectral beam combining systems are the two alternatively brightness-maintained approaches to break through the power scaling limitations of the monolithic fiber laser/amplifier ${ }^{[7-10]}$. In coherent and spectral beam combining systems, high brightness fiber amplifier with characteristic of narrow bandwidth is their

Correspondence to: P. Ma and P. Zhou, College of Advanced Interdisciplinary Studies, National University of Defense Technology, Changsha 410073, China. Email: zhoupu203@163.com (P. Zhou);

shandapengfei@126.com (P. Ma). basic unit, which tightly determines the power scaling ability of the beam combining systems. Traditional studies show that output power of this type of fiber source is mainly restricted by the effects of nonlinear stimulated Brillouin scattering (SBS) and/or TMI ${ }^{[4-6]}$.

As for SBS effect, some effective approaches had been proposed to mitigate its influence over the past decade. These approaches mainly include incorporating thermal and longitudinal stress gradients ${ }^{[11-13]}$, using counterpropagating pump ${ }^{[14]}$, special transverse-designed active fiber $^{[12,15]}$, multi-longitudinal-mode fiber oscillator ${ }^{[16,17]}$, filtered super-fluorescent source ${ }^{[10,18]}$, multi-wavelength injection and laser gain competition ${ }^{[19,20]}$ and applying phase modulation technique ${ }^{[8,9,20-29]}$. Within these SBS management approaches, phase modulation technique is a preferable approach beyond the output power of multikilowatt level ${ }^{[22-29]}$. As for TMI effect, several potential 
suppressing methods had also been proposed and validated by investigating on its physical mechanism ${ }^{[30-37]}$. Generally, TMI effect could be suppressed by optimizing the doping area or imposing external bend loss of higher-order modes for mode selection ${ }^{[23-26,30-33]}$, reducing the core size or numerical aperture (NA) of the active fiber ${ }^{[27,28,31,34]}$, increase of the seed power ${ }^{[33]}$, weakening head load by optimizing the injected ratios of co-pumped and counterpumped laser ${ }^{[33,35,36]}$, decrease of quantum defect and/or pump absorption ${ }^{[33,35,37]}$. Nowadays, as high as $3 \mathrm{~kW}$ and $3.5 \mathrm{~kW}$ output powers with narrow bandwidths and near-diffraction-limited beam qualities had been respectively presented by using gold-coated specialty gain fiber ${ }^{[24]}$ and low-NA active fiber ${ }^{[28]}$, while the configurations are not allfiberized and some bulk space components were employed. As for all-fiberized formats, as high as $\sim 2.5 \mathrm{~kW}$ power level had been reported both in nonpolarization-maintained and polarization-maintained fiber amplifiers ${ }^{[25,26,29]}$.

Despite most of the high power, narrow-bandwidth fiber amplifiers being pumped by $976 \mathrm{~nm}$ laser diodes (LDs) for being favorable of SBS management, tandem pumping is a robust route for simultaneously decreasing the quantum defect and pump absorption for TMI suppression ${ }^{[33,37]}$. This technique had been widely used in high power fiber amplifiers with broadband spectral characteristics ${ }^{[38-42]}$, and the output powers had been remarkably scaled to be beyond $3.5 \mathrm{~kW}$ power level by several groups ${ }^{[38,41,42]}$. Due to the small absorption area of the Yb-doped active fiber at $1018 \mathrm{~nm}$, one point should be noted that the suppressions of SBS and stimulated Raman scattering (SRS) are more difficult when tandem pumping is employed in high power, narrow-bandwidth fiber amplifiers.

In this manuscript, we tentatively introduce tandem pumping strategy for TMI suppression in a $4 \mathrm{~kW}$ power level narrow-bandwidth and all-fiberized amplifier. In order to further suppress SBS effect, one-stage phase modulation with large amplitude sinusoid or white noise signals (WNSs) is employed. SBS limited output powers of $336 \mathrm{~W}, 1.2 \mathrm{~kW}$ and $3.94 \mathrm{~kW}$ are obtained with spectral bandwidths accounting for $90 \%$ power of $\sim 0.025,0.18$ and $\sim 0.89 \mathrm{~nm}$, respectively. Compared with our previous $976 \mathrm{~nm}$ pumping system, TMI threshold is overall enhanced to be $>5$ times and the contribution of tandem pumping could be as high as $>3$ times. The residual TMI threshold enhancement is contributed to the increase of the seed power. The $M^{2}$ factor is measured to be within 1.5 below the TMI threshold and it is ultimately saturated to be 1.86 with the impact of TMI effect.

\section{Experimental setup}

The experimental setup of the high power tandem-pumped, narrow-bandwidth and all-fiberized fiber amplifier is shown in Figure 1, which is based on a typical master oscillator power amplification (MOPA) format. The seed laser (Seed) is a low noise, strictly single-frequency (linewidth $<20 \mathrm{kHz}$ ) fiber laser constructed by an ultrashort cavity with high gain phosphate glass active fiber ${ }^{[43]}$. The output power of the Seed is measured to be $60 \mathrm{~mW}$ with central wavelength of $1064.4 \mathrm{~nm}$. First, the spectral bandwidth of the seed laser is broadened by using one-stage phase modulation system for SBS suppression, which consists of a signal generator and a phase modulator (PM). In the experiment, large amplitude sinusoid or filtered WNSs with different electronic bandwidths and modulation depths are alternatively employed. The insertion loss of the PM is about $3 \mathrm{~dB}$ and the output power after PM is $\sim 30 \mathrm{~mW}$. After $\mathrm{PM}$, the laser power is pre-amplified by using three-stage all-fiberized pre-amplifiers (P-AI, P-AII, P-AIII). P-AI is a core-pumped pre-amplifier with $\sim 0.8 \mathrm{~m}$ highly doped (pump absorption coefficient $\sim 250 \mathrm{~dB} / \mathrm{m} @ 976 \mathrm{~nm}$ ), single-clad Yb-doped active fiber (YDF, core/cladding diameter ratio of $\sim 6 / 125 \mu \mathrm{m}$ ). LD1 with maximal output power of $480 \mathrm{~mW}$ and central wavelength of $976 \mathrm{~nm}$ is employed to pump the active fiber via a wavelength division multiplexer (WDM). The output power could be scaled to $\sim 150 \mathrm{~mW}$ after an isolator (ISO1) to block off the backward power. P-AII and P-AIII are two cladding-pumped pre-amplifiers. As for P-AII, LD2 with maximal output power of $\sim 9 \mathrm{~W}$ and central wavelength of $974 \mathrm{~nm}$ is used to pump $3.5 \mathrm{~m}$ long active fiber (YDF-I, pump absorption coefficient $\sim 4 \mathrm{~dB} / \mathrm{m}$ @ $975 \mathrm{~nm})$ via a $(2+1) \times 1$ combiner. The core and cladding diameters of the active fiber are $10 \mu \mathrm{m}$ and $125 \mu \mathrm{m}$, respectively. After P-AII, the output power is boosted to $\sim 3 \mathrm{~W}$ after ISO2. For P-AIII, the active fiber (YDF-II) is pumped by using two wavelength-stabilized laser diodes (LD3, LD4) with maximal output power of $\sim 90 \mathrm{~W}$ and central wavelength of $976 \mathrm{~nm}$ via a $(2+1) \times 1$ combiner. The core and cladding diameters of the active fiber are $15 \mu \mathrm{m}$ and $130 \mu \mathrm{m}$, respectively, and $2.8 \mathrm{~m}$ long active fiber with pump absorption coefficient of $\sim 5.4 \mathrm{~dB} / \mathrm{m} @ 976 \mathrm{~nm}$ is used. After P-AIII, the output power is further scaled to $\sim 70 \mathrm{~W}$ after ISO3. At the rear end port of P-AIII, a fiber coupler (C) is incorporated into the MOPA system. The coupling ratios of the out port of $\mathrm{C}$ to signal port and backward monitor (BM) port are $99.9 \%$ and $0.1 \%$, respectively. $0.1 \%$ port is used for diagnosing the SBS effect and ensuring the safety of the whole MOPA system during the power scaling process.

The final amplifier is pumped by using three $2 \mathrm{~kW}$ power level $1018 \mathrm{~nm}$ all-fiberized lasers via a $(6+1) \times 1$ combiner. Each $2 \mathrm{~kW}$ level $1018 \mathrm{~nm}$ pump laser is constructed by power combining of seven $300 \mathrm{~W}$ level $1018 \mathrm{~nm}$ single mode fiber lasers via a homemade $7 \times 1$ all-fiberized power combiner ${ }^{[44]}$. The active fiber used in this amplifier is large mode area and double clad Yb-doped fiber (YDF-III) with a core diameter of $30 \mu \mathrm{m}$ and an inner cladding diameter of $250 \mu \mathrm{m}$. The pump absorption coefficient is measured to 


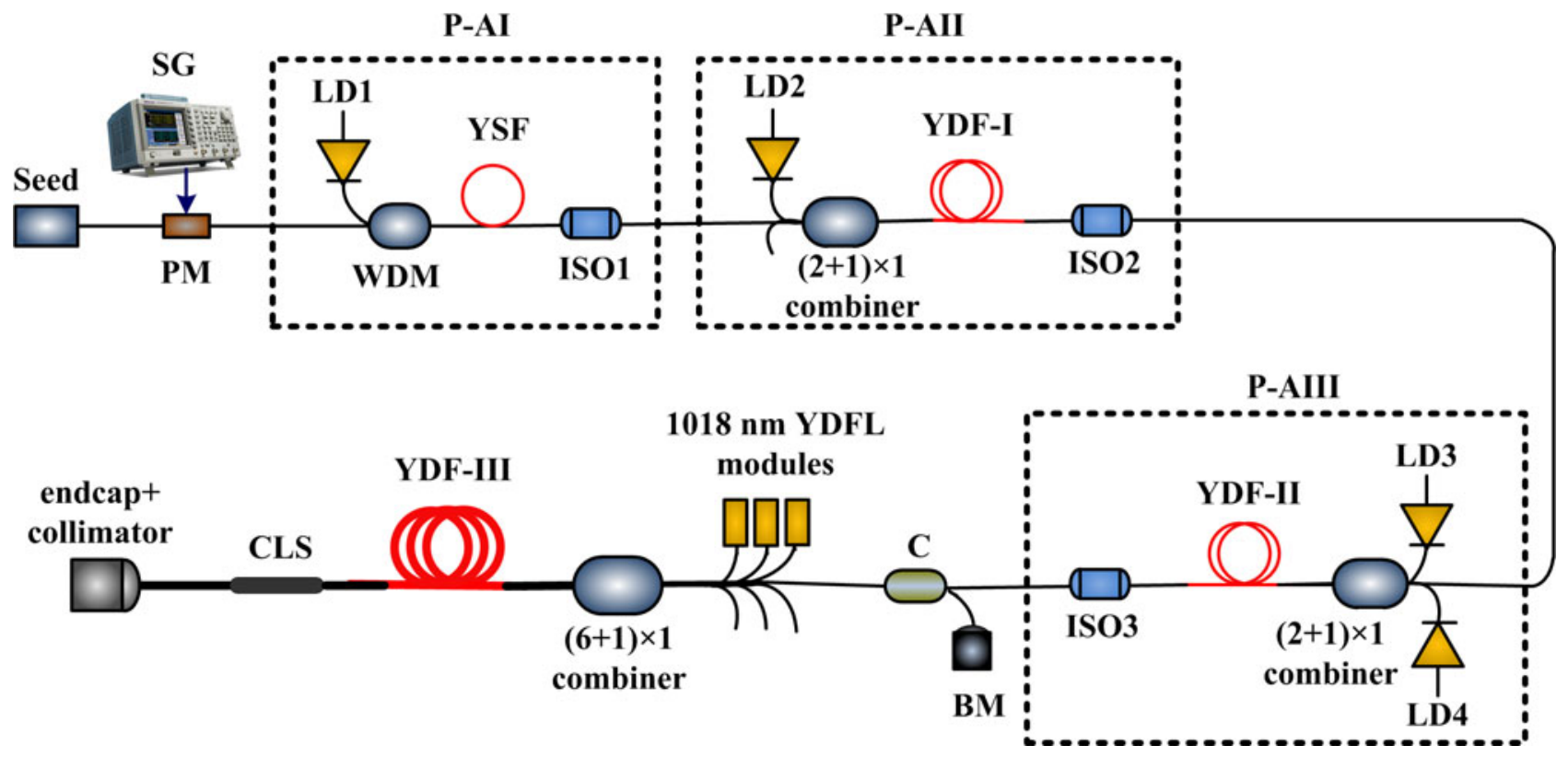

Figure 1. Experimental setup of high power tandem-pumped, narrow-bandwidth and all-fiberized fiber amplifier.

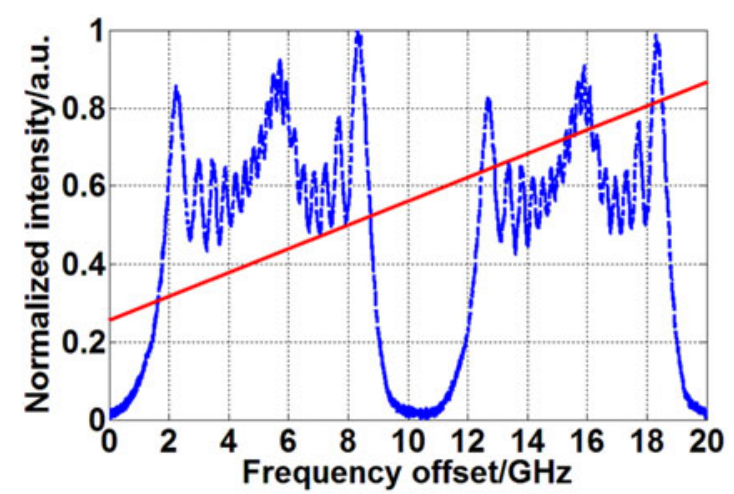

(a)

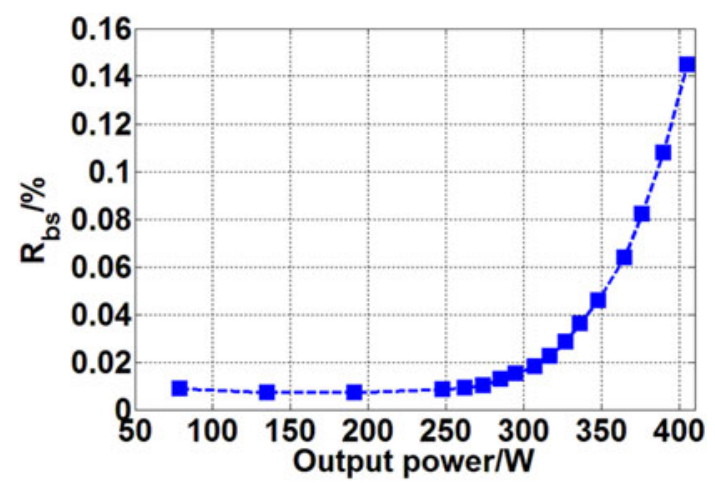

(b)

Figure 2. (a) The measured spectral distribution of the phase modulated laser; (b) the trend of backward power ratio $\left(R_{b s}\right)$ along with power scaling.

be $\sim 0.5 \mathrm{~dB} / \mathrm{m} @ 1018 \mathrm{~nm}$ and $\sim 31 \mathrm{~m}$ long active fiber is employed for high power amplification. The amplified laser is successively delivered through homemade cladding light stripper (CLS), fiber endcap and collimator to free space.

\section{Experimental results and discussions}

\subsection{Power scaling of large amplitude sinusoid signal modulated seed}

For achieving the criteria of SBS threshold at relatively safety state, we first investigate the power scaling process and SBS suppressing effect by using large amplitude sinusoid signal for phase modulation. The amplitude and repetition rate of the phase modulated signal are set to be $50 \mathrm{~V}$ and $100 \mathrm{MHz}$, respectively. The typical half-wave voltage and electro-optic bandwidth within $1 \mathrm{~dB}$ of the PM used in this section are $2 \mathrm{~V} @ 50 \mathrm{kHz}$ and $150 \mathrm{MHz}$, respectively. The spectral distribution of the phase modulated laser is shown in Figure 2(a) and the trend of backward power ratio $\left(R_{b s}\right)$ along with power scaling is shown in Figure 2(b). The spectral distribution is measured by using a FabryPerot interferometer (FPI) with free spectral range (FSR) of $10 \mathrm{GHz}$ and resolution limit of about $\sim 68 \mathrm{MHz} . \quad R_{b s}$ is defined as the ratio of actual backward power to the output power. The actual backward power is calculated by dividing the backward coupling ratio $(0.1 \%)$ by the collected power values of BM. As shown in Figure 2(a), the spectral range is well within $10 \mathrm{GHz}$ by using large amplitude sinusoid signal modulation. Since the spectral distribution by phase modulation is normally irregular, the spectral range accounting for $90 \%$ power is defined as the spectral bandwidth in all the following cases. This spectral 


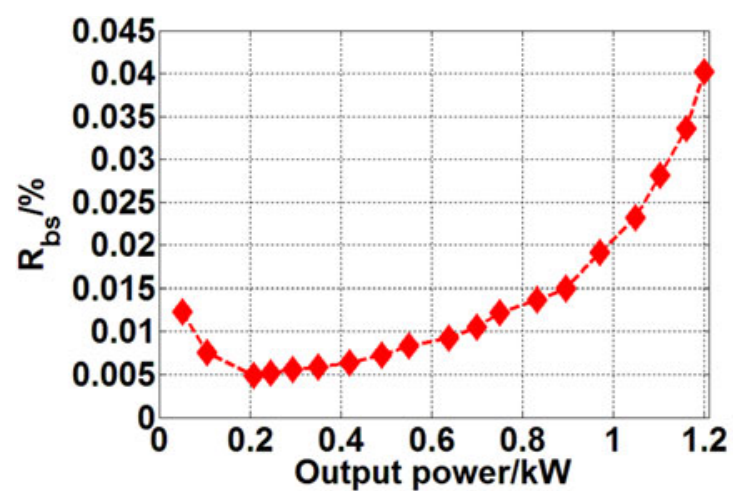

(a)

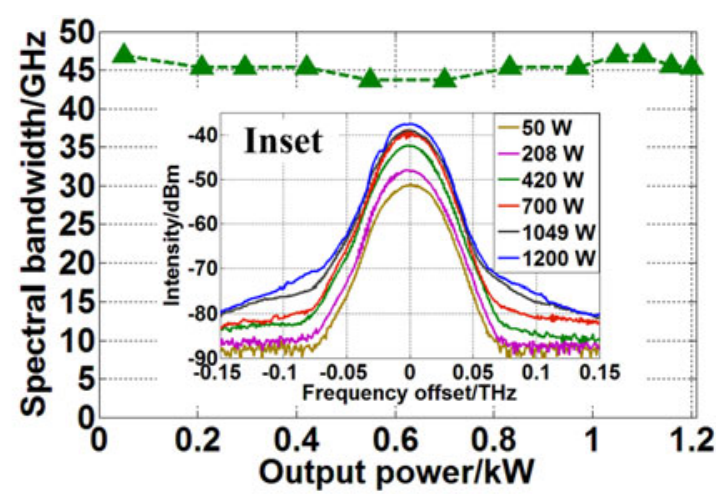

(b)

Figure 3. (a) The change of $R_{b s}$ along with output power; (b) the spectral bandwidth as a function of output power (inset: the frequency offset distributions at different output powers).

bandwidth criterion is conservative for both coherent and spectral beam combining systems design. By calculation, the spectral bandwidth is about $6.6 \mathrm{GHz}(0.025 \mathrm{~nm})$. As shown in Figure 2(b), when the output power is beyond $\sim 336 \mathrm{~W}$, $R_{b s}$ exponentially increases along with power scaling, which corresponds to an $R_{b s}$ inflection value of $\sim 0.04 \%$. This $R_{b s}$ inflection value is defined as the criteria of SBS threshold in the following experiments.

\subsection{Power scaling of filtered $2 \mathrm{GHz}-W N S$ modulated seed}

In this section, we investigate the power scaling process and SBS suppressing effect by using filtered WNS signal with $2 \mathrm{GHz}$ electronic bandwidth for phase modulation. The broadband electronic WNS signal is first filtered by using an electronic low-pass filter and then amplified to an amplitude of $4.5 \mathrm{~V}$ for phase modulation and spectral broadening. The typical half-wave voltage of the PM used in this case is $2.7 \mathrm{~V}$ (a) $1 \mathrm{GHz}$, and the electro-optic bandwidth at $2 \mathrm{~dB}$ is about $15 \mathrm{GHz}$. The increase of $R_{b s}(\%)$ along with power scaling process is shown in Figure 3(a), which shows that SBS limited output power of $\sim 1.2 \mathrm{~kW}$ could be achieved in this case. Figure 3(b) shows the change of spectral bandwidth as a function of output power scaling, and the inset in Figure 3(b) gives the frequency offset $(\mathrm{THz})$ distributions of the amplified laser at several different output powers. As shown in Figure 3(b), the spectral bandwidth broadens little along with the increase of the output power. Inset shown in Figure 3(b) denotes that Gaussian-like spectra with well symmetry are generated by the spectral broadening system. The measured spectral bandwidth is just $\sim 45 \mathrm{GHz}$ $(\sim 0.17 \mathrm{~nm})$ at $1.2 \mathrm{~kW}$ power level. Besides, by analysis, the full width at half maximum (FWHM) of the spectral range is also broadened little with the increase of the output power. In the experiment, the essential reason for avoiding spectral broadening is that the temporal characteristic of the single-frequency seed laser is quite stable and self-phasemodulation effect induced noise transfer from intensity to phase is weak $^{[45]}$. At the maximal output power, the FWHM of the spectral range is $\sim 31 \mathrm{GHz}(\sim 0.12 \mathrm{~nm})$.

\subsection{Power scaling of filtered $12 \mathrm{GHz}-W N S$ modulated seed}

For further SBS management, an electronic low-pass filter with $12 \mathrm{GHz}$ electronic bandwidth is used for phase modulation and spectral broadening. The filtered WNS is amplified to an amplitude of $5 \mathrm{~V}$. The type of PM used in this situation is identical as shown in Section 3.2. Figure 4(a) shows the power scaling process of the MOPA system along with the increase of pump power, which indicates a slope efficiency of $78 \%$ and a well linear-fitting trend. With injected pump power of $5057 \mathrm{~W}, 3940 \mathrm{~W}$ output power is ultimately achieved. The increase of $R_{b s}(\%)$ as a function of the output power is shown in Figure 4(b), which denotes that SBS threshold is about $\sim 3.9 \mathrm{~kW}$. At $3940 \mathrm{~W}$, the backward power ratio $\left(R_{b s}\right)$ is measured to be $0.046 \%$.

With the increase of output power, the change of spectral bandwidth is shown in Figure 5(a) and the frequency offset (THz) of the emitted laser at different output powers is shown in the inset of Figure 5(a). From Figure 5(a), it could be inferred that the spectral bandwidth fluctuates between $0.245 \mathrm{THz}$ and $0.205 \mathrm{THz}$ in the whole power scaling process. The fluctuation phenomenon of spectral bandwidth is attributed to the spectral asymmetry of the amplified laser, as shown in the inset of Figure 5(a). The spectral asymmetry of the amplified laser is mainly caused by the comprehensive effects of limited electro-optic bandwidth of the PM and gain competitions in the whole amplifications. The sideband broadening is contributed to self-phase-modulation and four-wave-mixing effects ${ }^{[45,46]}$. At the maximal output power, the spectral bandwidth is measured to be $0.237 \mathrm{THz}(0.89 \mathrm{~nm})$. It should be noted that the time-coherence control of such a spectral bandwidth had been successfully fulfilled in $2 \mathrm{~kW}$ level coherent beam combining structure ${ }^{[8]}$. Compared with the spectral bandwidth $(0.245 \mathrm{THz})$ of the injected seed $(50 \mathrm{~W}$ power 


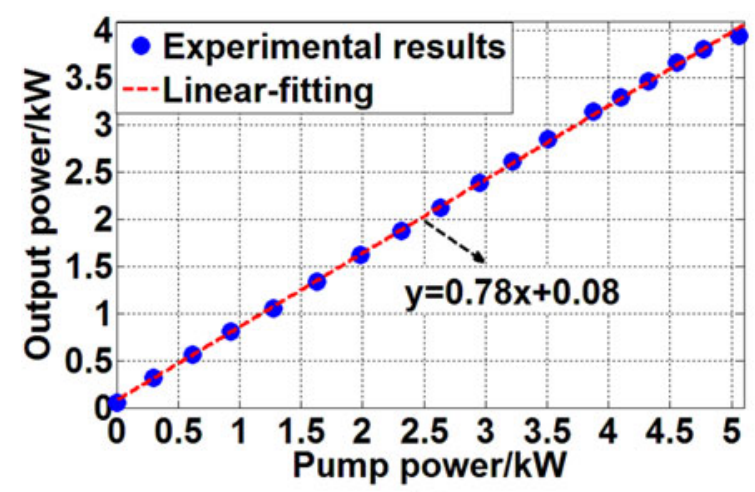

(a)

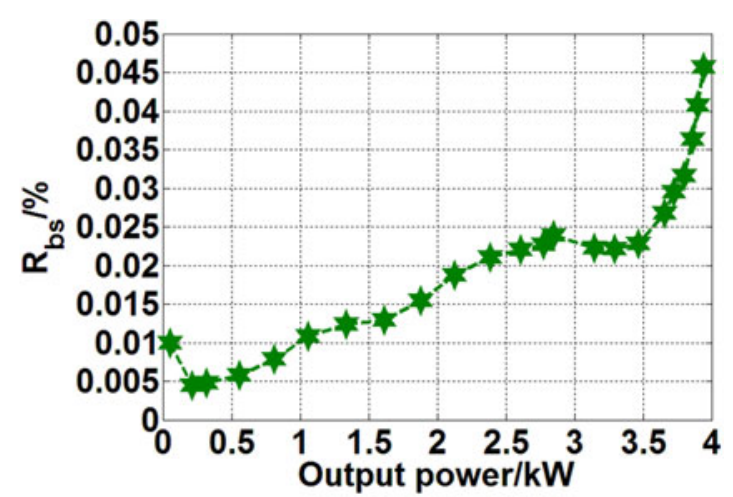

(b)

Figure 4. (a) The power scaling process of the MOPA system along with increase of pump power; (b) the increase of $R_{b s}(\%)$ as a function of the output power.

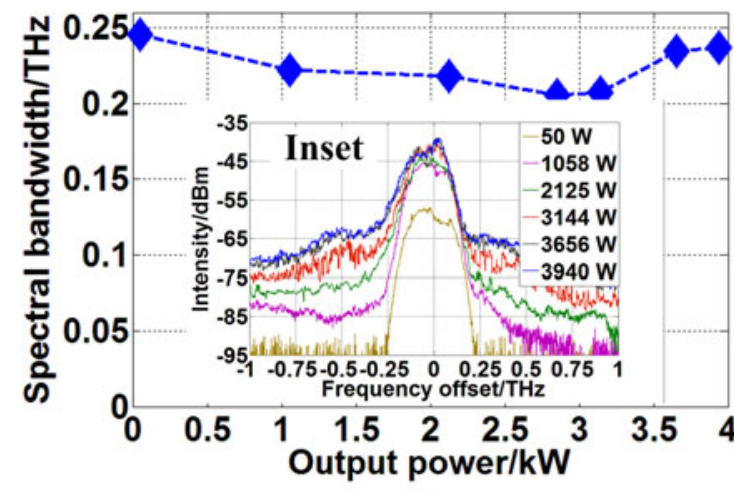

(a)

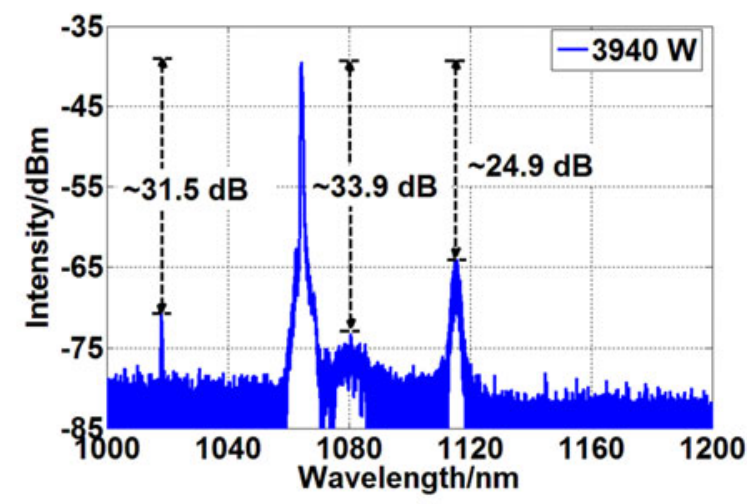

(b)

Figure 5. (a) The change of spectral bandwidth with the output power (inset: the frequency offset at several different output powers); (b) the spectrum from 1000 to $1200 \mathrm{~nm}$ at $3940 \mathrm{~W}$.

level), it broadens little when the output power boosts to be 3940 W. Figure 5(b) is the spanned emission spectrum from 1000 to $1200 \mathrm{~nm}$ at $3940 \mathrm{~W}$. The signal to noise ratio (SNR) of the signal laser is $\sim 31.5 \mathrm{~dB}, \sim 33.9 \mathrm{~dB}$ and $\sim 24.9 \mathrm{~dB}$ compared with the residual $1018 \mathrm{~nm}$ pump laser, amplified spontaneous emission and Raman Stokes light, respectively. Except for SBS effect, SRS will be another challenge for further power scaling of such high power, tandem-pumped fiber laser systems.

Along with power scaling, the intensity signal and the corresponding Fourier spectral distribution are diagnosed by using an oscilloscope with $1 \mathrm{GHz}$ bandwidth and an InGaAs photo-detector (PD) with $150 \mathrm{MHz}$ electro-optic bandwidth. In the experiment, when the output power scales from $2205 \mathrm{~W}$ to $2253 \mathrm{~W}$, a sudden intensity noise is observed in the PD-captured intensity distribution. The normalized intensity signals collected by the PD at $2205 \mathrm{~W}, 2253 \mathrm{~W}$ and $3940 \mathrm{~W}$ are depicted in Figure 6(a). By analysis, the standard deviations of the intensity signals are jumped from $1.38 \%$ to $2.5 \%$ when the output power increases from $2205 \mathrm{~W}$ to $2253 \mathrm{~W}$. The standard deviation of the intensity signal is saturated to be $2.8 \%$ at $3940 \mathrm{~W}$. Figure 6(b) shows the corresponding Fourier spectral distributions of the intensity signals at $2205 \mathrm{~W}, 2253 \mathrm{~W}$ and $3940 \mathrm{~W}$. Compared with the Fourier spectral distribution at output power of $2205 \mathrm{~W}$, noise-like protuberance exists within the frequency range from 0 to $5 \mathrm{kHz}$ at output power of $2253 \mathrm{~W}$. At $3940 \mathrm{~W}$, the frequency range of noise-like protuberance is extended from 0 to $\sim 20 \mathrm{kHz}$. By combining temporal-spectral characteristics of $\mathrm{TMI}^{[47,48]}$, it is confirmed that the TMI effect occurs when the output power is beyond $2253 \mathrm{~W}$. Further, the beam quality ( $M^{2}$ factor) of the amplified laser is examined along with power enhancement, which is shown in Figure 6(c). From Figure 6(c), it is concluded that the $M^{2}$ factor is well within 1.5 below the TMI threshold. The $M^{2}$ factor is transited to 1.55 at $2253 \mathrm{~W}$ and abruptly deteriorates to 1.88 at $2384 \mathrm{~W}$. For further power scaling, the $M^{2}$ factor is nearly maintained and saturated and it is measured to be 1.86 at 3940 W. Figure 6(d) shows the typical far-field beam profiles at different output powers. From Figure 6(d), it can be seen that the beam profile is near Gaussian-like with an output power of $1878 \mathrm{~W}$ (below TMI threshold). At TMI threshold $(2253 \mathrm{~W})$, some extent of distortion could be observed. For further power scaling to $2384 \mathrm{~W}, 2775 \mathrm{~W}$ and $3000 \mathrm{~W}$, the beam profiles are distorted seriously and obvious higherorder modes could be observed. For further power scaling to $3940 \mathrm{~W}$, the TMI has been transmitted to chaotic state and 


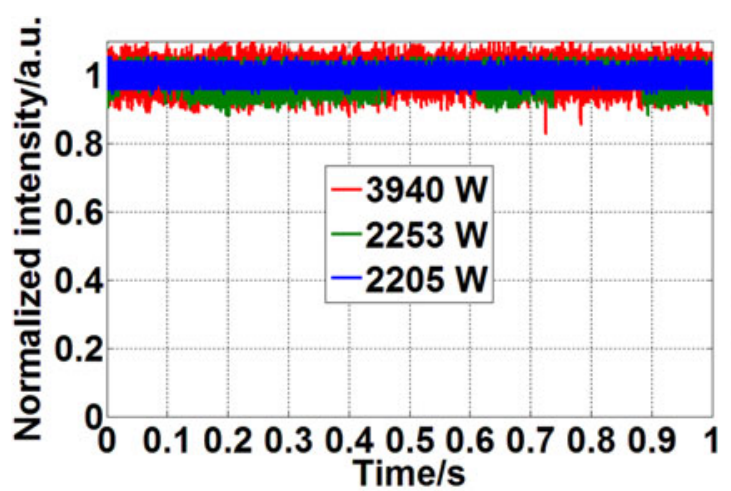

(a)

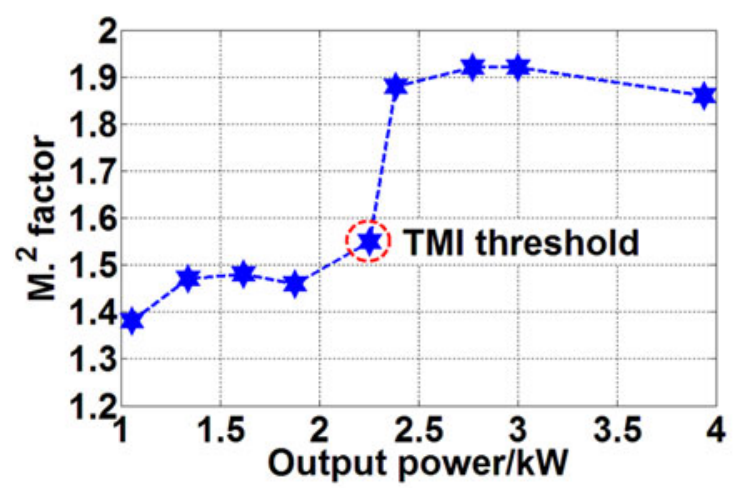

(c)

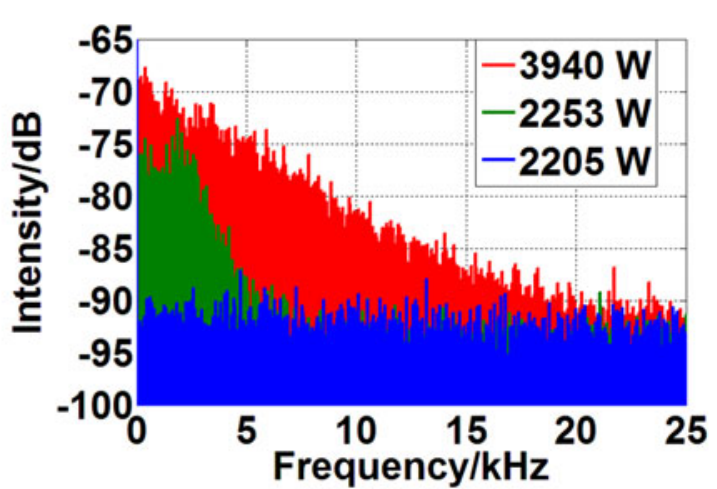

(b)

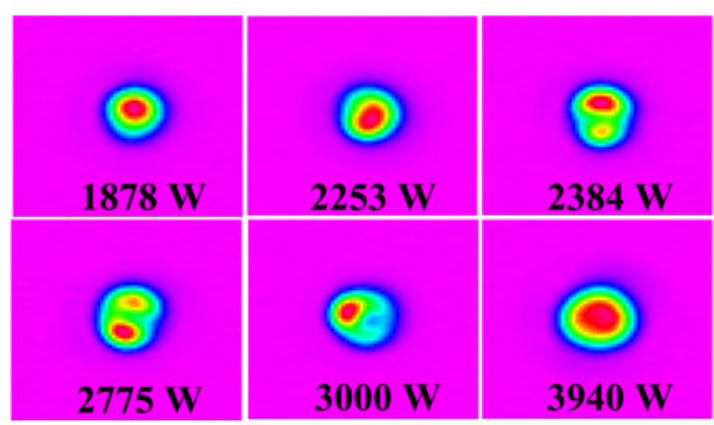

(d)

Figure 6. (a) The normalized intensity signals collected by PD; (b) the corresponding Fourier spectral distributions; (c) the measured $M^{2}$ factor along with power enhancement; (d) the typical far-field beam profiles at different output powers.

the beam profile is the superposition of fundamental mode and higher-order modes.

As shown above, the TMI threshold is measured to be $\sim 2253 \mathrm{~W}$ by using tandem pumping strategy. This TMI threshold is higher than 5 times compared with our previous result (TMI threshold $\sim 446 \mathrm{~W}$ ) in which the identical type of active fiber was pumped by $976 \mathrm{~nm}$ LDs and $\sim 10 \mathrm{~W}$ preamplified seed power was injected into the main amplifier for final power amplification ${ }^{[49]}$. By theoretical analysis based on a self-built model ${ }^{[33]}, 1018 \mathrm{~nm}$ tandem pumping manner could scale the TMI threshold of 3.2 times compared with $976 \mathrm{~nm}$ pumping case. Increase of the injected power from $10 \mathrm{~W}$ to $70 \mathrm{~W}$ could scale the TMI threshold of 1.4 times. By employing tandem-pumping-suitable active fiber for higher TMI threshold at $976 \mathrm{~nm}$, higher output power with neardiffraction-limited beam quality could be expected.

\section{Conclusions}

In this work, we demonstrate a record $4 \mathrm{~kW}$ power level all-fiberized, narrow-bandwidth MOPA system by using tandem pumping for TMI management. The SBS effect is balanced by using one-stage phase modulation technique for moderate spectral broadening. The SBS suppressing effects of large amplitude sinusoid signal and filtered WNSs are investigated. By defining the spectral offset range accounting for $90 \%$ power as the spectral bandwidth, SBS limited output powers of $336 \mathrm{~W}, 1.2 \mathrm{~kW}$ and $3.94 \mathrm{~kW}$ are respectively achieved with bandwidth of $\sim 0.025 \mathrm{~nm}$ (6.6 GHz), $0.17 \mathrm{~nm}(45 \mathrm{GHz})$ and $\sim 0.89 \mathrm{~nm}(0.237 \mathrm{THz})$. The TMI suppressing ability of this MOPA system is compared with our previous $976 \mathrm{~nm}$ pumped format, which indicates that TMI threshold is scaled to $>5$ times. By analysis, the $1018 \mathrm{~nm}$ tandem pumping could boost the threshold of $\sim 3.2$ times and the increase of injected seed power could enhance the threshold of 1.4 times. The $M^{2}$ factor of the amplified laser is well within 1.5 below the TMI threshold and it is ultimately saturated to be 1.86 with the impact of TMI at 3940 W. Further output power scaling is limited by the comprehensive effects of SBS, TMI and SRS, which will provide a well platform for investigating the mutual-coupled effects among the above three detrimental factors and the possible optimizing strategies for higher power, narrow-bandwidth fiber amplifiers.

\section{Acknowledgements}

This work is sponsored by the National Natural Science Foundation of China (Nos. 61705264 and 61705265). The authors would like to express sincere thanks to Liang Xiao, 
Jiawei He, Xiaoyong Xu, and Minyang Liu for their help in the experiment.

\section{References}

1. D. J. Richardson, J. Nilsson, and W. A. Clarkson, J. Opt. Soc. Am. B 27, B63 (2010).

2. M. N. Zervas and C. A. Codemard, IEEE J. Sel. Top. Quantum Electron. 20, 0904123S (2014).

3. W. Shi, Q. Fang, X.-S. Zhu, R. Norwood, and N. Peyghambarian, Appl. Opt. 53, 6554 (2014).

4. J. W. Dawson, J. M. Messerly, R. J. Beach, M. Y. Shverdin, E.A. Stappaerts, A. K. Sridharan, P. H. Pax, J. E. Heebner, C. W. Siders, and C. P. J. Barty, Opt. Express 16, 13240 (2008).

5. H. Otto, C. Jauregui, J. Limpert, and A. Tünnermann, Proc. SPIE 9728, 97280E (2016).

6. M. Zervas, Proc. SPIE 10512, 1051205 (2018).

7. T. Y. Fan, IEEE J. Sel. Top. Quantum Electron. 11, 567 (2005).

8. Z.-J. Liu, P.-F. Ma, R.-T. Su, R.-M. Tao, Y.-X. Ma, X.-L. Wang, and P. Zhou, J. Opt. Soc. Am. B. 34, A7 (2016).

9. I. Dajani, A. Flores, R. Holten, B. Anderson, B. Pulford, and T. Ehrenreich, Proc. SPIE 9728, 972801 (2016).

10. Y. Zheng, Y.-F. Yang, J.-H. Wang, M. Hu, G.-B. Liu, X. Zhao, X.-L. Chen, K. Liu, C. Zhao, B. He, and J. Zhou, Opt. Express 24, 12064 (2016).

11. L. Huang, H.-S. Wu, R.-X. Li, L. Li, P.-F. Ma, X.-L. Wang, J.-Y. Leng, and P. Zhou, Opt. Lett. 42, 1 (2017).

12. C. Robin, I. Dajani, and B. Pulford, Opt. Lett. 39, 666 (2014).

13. L. Zhang, S.-Z. Cui, C. Liu, J. Zhou, and Y. Feng, Opt. Express 21, 5456 (2013).

14. T. Theeg, H. Sayinc, J. Neumann, and D. Kracht, IEEE Photon. Tech. Lett. 24, 1864 (2012).

15. S. Gray, A. Liu, D. Walton, J. Wang, M. Li, X. Chen, A. Ruffin, J. Demeritt, and L. Zenteno, Opt. Express 15, 17044 (2007).

16. Y. Xu, Q. Fang, Y.-G. Qin, X.-J. Meng, and W. Shi, Appl. Opt. 54, 9419 (2015).

17. Z.-H. Huang, X.-B. Liang, C.-Y. Li, H.-H. Lin, Q. Li, J.-J. Wang, and F. Jing, Appl. Opt. 55, 297 (2016).

18. J.-M. Xu, W. Liu, J.-Y. Leng, H. Xiao, S.-F. Guo, P. Zhou, and J.-B. Chen, Opt. Lett. 40, 2973 (2015).

19. P. Weßels, P. Adel, M. Auerbach, D. Wandt, and C. Fallnich, Opt. Express 12, 4443 (2004).

20. N. Naderi, A. Flores, B. Anderson, and I. Dajani, Opt. Lett. 41, 3964 (2016).

21. A. V. Harish and J. Nilsson, IEEE J. Sel. Top. Quantum Electron. 24, 5100110 (2018).

22. A. Flores, C. Robin, A. Lanari, and I. Dajani, Opt. Express 22, 17735 (2014).

23. P.-F. Ma, R.-M. Tao, R.-T. Su, X.-L. Wang, P. Zhou, and Z.-J. Liu, Opt. Express 24, 4187 (2016).

24. C. X. Yu, O. Shatrovoy, T. Y. Fan, and T. F. Taunay, Opt. Lett. 41, 5202 (2016).

25. M. Kanskar, J. Zhang, J. Koponen, O. Kimmelma, V. Aallos, I-Ning Hu, and A. Galvanauskas, Proc. SPIE 10512, 105120F (2018).
26. R.-T. Su, R.-M. Tao, X.-L. Wang, H.-W. Zhang, P.-F. Ma, P. Zhou, and X.-J. Xu, Laser Phys. Lett. 14, 085102 (2017).

27. K. Brar, M. Savage-Leuchs, J. Henrie, S. Courtney, C. Dilley, R. Afzal, and E. Honea, Proc. SPIE 8961, 89611R (2014).

28. F. Beier, C. Hupel, S. Kuhn, S. Hein, J. Nold, F. Proske, B. Sattler, A. Liem, C. Jauregui, J. Limpert, N. Haarlammert, T. Schreiber, R. Eberhardt, and A. Tünnermann, Opt. Express 25, 14892 (2017).

29. N. Platonov, R. Yagodkin, J. D. L. Cruz, A. Yusim, and V. Gapontsev, Proc. SPIE 10512, 105120E (2018).

30. A. V. Smith and J. J. Smith, Opt. Express 19, 10180 (2011).

31. B. Ward, C. Robin, and I. Dajani, Opt. Express 20, 11407 (2012).

32. M. Laurila, M. Jørgensen, K. Hansen, T. Alkeskjold, J. Broeng, and J. Lægsgaard, Opt. Express 20, 5742 (2012).

33. R.-M. Tao, X.-L. Wang, and P. Zhou, IEEE J. Sel. Top. Quantum Electron. 24, 0903319 (2018).

34. C. Jauregui, T. Eidam, H. Otto, F. Stutzki, F. Jansen, J. Limpert, and A. Tünnermann, Opt. Express 20, 12912 (2012).

35. A. Smith and J. Smith, Opt. Express 21, 15168 (2013).

36. Z. Eznaveh, G. Galmiche, E. Lopez, and R. Correa, Proc. SPIE 9344, 93442G (2015).

37. C. Jauregui, H. Otto, F. Stutzki, F. Jansen, J. Limpert, and A. Tünnermann, Opt. Express 21, 19375 (2013).

38. D. J. Richardson, J. Nilsson, and W. A. Clarkson, J. Opt. Soc. Am. B 27, B63 (2010).

39. C. Wirth, O. Schmidt, A. Kliner, T. Schreiber, R. Eberhardt, and A. Tünnermann, Opt. Lett. 36, 3061 (2011).

40. H. Xiao, J.-Y. Leng, H.-W. Zhang, L. Huang, J.-M. Xu, and P. Zhou, Appl. Opt. 54, 8166 (2015).

41. P. Zhou, H. Xiao, J.-Y. Leng, J.-M. Xu, Z.-L. Chen, H.-W. Zhang, and Z.-J. Liu, J. Opt. Soc. Am. B. 34, A29 (2017).

42. P. Yan, X.-J. Wang, Z.-H. Wang, Y.-S. Huang, D. Li, Q.-R. Xiao, and M.-L. Gong, IEEE J. Sel. Top. Quantum Electron. 24, 0902506 (2018).

43. S.-H. Xu, Z.-M. Yang, W.-N. Zhang, X.-M. Wei, Q. Qian, D.-D. Chen, Q.-Y. Zhang, S.-X. Shen, M.-Y. Peng, and J.-R. Qiu, Opt. Lett. 36, 3708 (2011).

44. R.-X. Li, H. Xiao, J.-Y. Leng, Z.-L. Chen, J.-M. Xu, J. Wu, and P. Zhou, Laser Phys. Lett. 14, 125102 (2017).

45. W. Liu, W.-J. Kuang, M. Jiang, J.-M. Xu, P. Zhou, and Z.-J. Liu, Laser Phys. Lett. 13, 035105 (2016).

46. A. G. Kuznetsov, E. V. Podivilov, and S. A. Babin, J. Opt. Soc. Am. B 29, 1231 (2012).

47. H. Otto, F. Stutzki, F. Jansen, T. Eidam, C. Jauregui, J. Limpert, and A. Tünnermann, Opt. Express 20, 15710 (2012)

48. I.-N. Hu, C. Zhu, C. Zhang, A. Thomas, and A. Galvanauskas, Proc. SPIE 8601, 860109 (2013).

49. R.-M. Tao, P.-F. Ma, X.-L. Wang, P. Zhou, and Z.-J. Liu, J. Opt. 18, 065501 (2016). 